Artículo de investigación científica.

Cómo citar: M. Caicedo, P. Guzmán, A. Castro y K. Robayo. "Diseño hidrológico de un sistema VAC para la finca el Caimo ubicada en el municipio de Villa de Leyva, Boyacá". Inventum, vol. 15, no. 29 , pp. 59-75. doi: 10.26620/uniminuto. inventum.15.29.2020.59-75

Editorial: Corporación Universitaria Minuto de Dios - UNIMINUTO.

ISSN: $1909-2520$

elSSN: $2590-8219$

Fecha de recibido: 31 de mayo de 2020

Fecha de aprobado: 15 de junio de 2020

Fecha de publicación: 15 de julio de 2020

Conflicto de intereses: los autores han declarado que no existen intereses en competencia.

Manuela Caicedo Garzón

mcaicedoga@unbosque.edu.co

https://orcid.org/0000-0003-0740-9119

Universidad El Bosque, Bogotá

Colombia

Paula Alejandra Guzmán Parra pguzmanp@unbosque.edu.co https://orcid.org/0000-0003-4364-0887 Universidad El Bosque, Bogotá Colombia

Valeria Andrea Castro Hernández vcastroh@unbosque.edu.co https://orcid.org/0000-0001-6811-7094 Universidad El Bosque, Bogotá Colombia

Karen Daniela Robayo Martínez krobayom@unbosque.edu.co https://orcid.org/0000-0003-4136-7004 Universidad El Bosque, Bogotá Colombia

\section{Diseño hidrológico de un sistema VAC para la finca el Caimo ubicada en el municipio de Villa de Leyva, Boyacá}

\author{
Hydrological design of a VAC system \\ for the El Caimo farm located in the \\ municipality of Villa de Leyva, Boyacá
}

\begin{abstract}
Resumen
Este artículo propone mejorar la productividad del agroecosistema de la finca El Caimo, ubicada en el municipio de Villa de Leyva, Boyacá, al rehabilitar su sistema de producción agrícola; con la implementación de un sistema agrícola integrado de cultivo que forma parte del Manual Básico de Agro-acuicultura integrada de la Organización de las Naciones Unidas para la Alimentación y la Agricultura (FAO). Se trata del sistema VAC de Vietnam del Norte, que integra los elementos que forman parte de su estructura, con ello se busca su funcionamiento y sinergia con la combinación de los elementos y su distribución en el espacio, sumado a cambios en la cobertura actual (uso del suelo) de la finca El Caimo. A partir de la elaboración de un diseño hidrológico, con el objeto de interpretar y cuantificar, mediante modelos matemáticos, los procesos naturales inmersos en la dinámica hidrológica que intervienen en el desarrollo productivo de la finca El Caimo, y de forma paralela la elaboración de un modelo de elevación digital (DEM) por medio de Sistemas de Información Geográfica (SIG) para la consulta de datos e información hidrometeorológica, y la representación de datos georreferenciados para su análisis con la utilización de un software, y así encontrar patrones de comportamiento de distintas variables, para la delimitación de la cuenca hidrográfica cercana a la finca, con el propósito de identificar puntos estratégicos para la captación natural del agua de precipitación y su descarga de agua por escurrimiento dirigida al abastecimiento natural de los componentes del sistema VAC, al tratarse de interacciones entre los sistemas climático y terrestre. Lo cual, resulta aplicable en el sistema de producción agrícola de la finca El Caimo, que se desarrolla en el manejo de un agroecosistema que requiere de la disponibilidad de recursos para su funcionamiento, entre ellos, la disponibilidad de agua y su aprovechamiento; al tratarse de un sistema socioeconómico complejo, limitado y determinado por el sistema social y político en el que se encuentra; por el valor que la economía asigna a los recursos y productos de la finca; por la falta de infraestructura vial, mano de obra, capital, tecnología e información que permite o limita la integración de los componentes que el predio ofrece, y que en conjunto
\end{abstract}


contribuyen al desarrollo del proceso de producción. Situación que explica su baja productividad, consecuencia de la falta de implementación de buenas prácticas agrícolas. De esta manera se obtiene un $4 \%$ de disminución en el potencial de escorrentía de la finca El Caimo, con un cambio en su cobertura actual, por una mayor cobertura vegetal en árboles con la siembra de especie endémica de roble andino (Quercus humboldtii) apoyada de la siembra de cultivos que frutales (mora, durazno) y hortalizas (papa) que se producen actualmente en la finca, y en concordancia con el aprovechamiento máximo de 1,475,823 L de agua de infiltración. Asimismo, el cálculo de disminución del caudal provocado por la nueva cobertura arbórea en la finca se determinó mediante el tránsito de crecientes, lo que indicó una reducción de $0,428 \mathrm{~m} 3 / \mathrm{h}$, y así favoreció la disposición del sistema VAC en la finca El Caimo.

Palabras clave: agroecosistema, cobertura, caudal, diseño hidrológico, dinámica hidrológica, Sistemas de información Geográfica (SIG), sistema VAC, tránsito de crecientes.

\begin{abstract}
His article proposes to improve the productivity of the agro-ecosystem of the El Caimo farm located in the municipality of Villa de Leyva, Boyacá, by rehabilitating its agricultural production system; implementing an integrated agricultural system of cultivation that is part of the Basic Manual of Integrated Agro-Aquaculture of the United Nations Food and Agriculture Organization (FAO), being the VAC system of North Vietnam, which integrates the elements that are part of its structure looking for its operation and synergy taking into account the combination of the elements and their distribution in space, added to changes in the current coverage (land use) of the El Caimo farm. This is based on the elaboration of a hydrological design, with the aim of interpreting and quantifying through mathematical models the natural processes immersed in the hydrological dynamics that intervene in the productive development of the El Caimo estate, and in parallel the elaboration of a digital elevation model (DEM) through Geographical Information Systems (GIS) for the consultation of data and hydrometeorological information and the representation of georeferenced data for its analysis through a software to find behaviour patterns of different variables, for the delimitation of the hydrographic basin near the farm, with the purpose of identifying strategic points for the natural capture of precipitation water and its discharge by runoff directed to the natural supply of the components of the VAC system, as these are interactions between the climate and land systems. This is applicable to the agricultural production system of the El Caimo farm, which is developed in the management of an agro-ecosystem that requires the availability of resources for its operation, including water availability and its use, since it is a complex socioeconomic system, limited and determined by the social and political system in which it is located; by the value that the economy assigns to the farm's resources and products; by the lack of road infrastructure, labor, capital, technology, and information that allows or limits the integration of the components that the farm offers, and that together contribute to the development of the production process. This situation explains its low productivity, which is a consequence of the lack of implementation of good agricultural practices. In this way, a $4 \%$ decrease in the run-off potential of the El Caimo farm is obtained
\end{abstract}


with a change in its current coverage, due to a greater plant cover in trees with the planting of endemic species of Andean Oak (Quercus humboldtii) supported by the planting of crops that are currently produced on the farm, such as fruit (blackberry, peach) and vegetables (potato), and in accordance with the maximum use of 1,475,823 L of infiltration water, also the calculation of the decrease in flow due to the new tree cover on the farm was determined by the transit of floods which indicated a reduction of $0.428 \mathrm{~m} 3 / \mathrm{h}$, which favours the availability of the VAC system on the $\mathrm{El}$ Caimo farm.

Keywords: agro-ecosystem, coverage, flow, hydrological design, hydrological dynamics, Geographical Information Systems (GIS), VAC system, transit of floods.

\section{Resumo}

Este artigo se propõe a melhorar a produtividade do agroecossistema da fazenda El Caimo localizada no município de Villa de Leyva, Boyacá, por meio da reabilitação de seu sistema de produção agrícola; implementar um sistema de agropecuária integrado que faz parte do Manual Básico de Agroaquicultura Integrada da Organização das Nações Unidas para Agricultura e Alimentação (FAO), no caso do sistema VAC do Vietnã do Norte, que integra os elementos que o compõem parte de sua estrutura buscando seu funcionamento e sinergia levando em consideração a combinação dos elementos e sua distribuição no espaço, somada a mudanças na atual cobertura (uso do solo) da Fazenda El Caimo. A partir da elaboração de um projeto hidrológico, com o objetivo de interpretar e quantificar através de modelos matemáticos os processos naturais imersos na dinâmica hidrológica que interferem no desenvolvimento produtivo da Fazenda El Caimo, e em paralelo a elaboração de um modelo digital de elevação (DEM) através de Sistemas de Informação Geográfica (SIG) para consulta de dados e informações hidrometeorológicas e representação de dados georreferenciados para análise através de software e assim encontrar padrões de comportamento de diferentes variáveis, para a delimitação da bacia hidrográfica próxima à fazenda, com o objetivo de identificar pontos estratégicos para a captação natural das águas pluviais e seu lançamento por escoamento visando o abastecimento natural dos componentes do sistema VAC, uma vez que são interações entre os clima e sistemas terrestres. Que se aplica ao sistema de produção agrícola da Fazenda El Caimo, que se desenvolve no manejo de um agroecossistema que requer a disponibilidade de recursos para seu funcionamento, entre eles, a disponibilidade de água e seu uso; por ser um sistema socioeconômico complexo, limitado e determinado pelo sistema social e político em que se encontra; pelo valor que a economia atribui aos recursos e produtos da fazenda; pela falta de infraestrutura viária, mão de obra, capital, tecnologia e informação que permita ou limite a integração dos componentes que a propriedade oferece, e que juntos contribuam para o desenvolvimento do processo produtivo. Situação que explica sua baixa produtividade, consequência da falta de implantação de boas práticas agrícolas. Desta forma, obtém-se uma redução de $4 \%$ no potencial de escoamento da fazenda El Caimo com a mudança de sua cobertura atual, devido a uma maior cobertura vegetal arbórea com o plantio de espécies endêmicas de carvalho andino (Quercus humboldtii) apoiado por a semeadura das lavouras de frutíferas (amora, pêssego) e 
hortaliças (batata) que hoje são produzidas na fazenda, e de acordo com a utilização máxima de $1.475 .823 \mathrm{~L}$ de água de infiltração, também o cálculo da diminuição da vazão devido à nova cobertura $\mathrm{A}$ área arbórea da fazenda foi determinada através do tráfego de enchentes, o que indicou uma redução de 0,428 $\mathrm{m} 3 / \mathrm{h}$, o que favorece a disponibilidade do sistema VAC na fazenda El Caimo.

Palavras-chave: agroecossistema, cobertura, fluxo, projeto hidrológico, dinâmica hidrológica, Sistemas de Informação Geográfica (SIG), sistema VAC, tráfego de inundação. 


\section{I.INTRODUCCIÓN}

Los diseños hidrológicos son un conjunto de análisis cuantitativos sobre fenómenos naturales que están relacionados con el ciclo hidrológico [1], estos tienen una gran importancia, ya que permiten mostrar y entender la disponibilidad de agua de un sector definido para poder dimensionar una correcta estructura en el funcionamiento de obras hidráulicas [2]. Dichos análisis fueron aplicados en la finca El Caimo en el municipio de Villa de Leyva, Boyacá, para juzgar el comportamiento de la cuenca aledaña con el objetivo de identificar la disponibilidad del recurso hídrico y poder aprovecharlo al máximo.

Para la realización de un diseño hidrológico, existen grandes obstáculos [2]. Latinoamérica no posee numerosa información pluviométrica, en consecuencia, los trabajos hidrológicos están basados mayoritariamente por datos de precipitación, y esto promueve cierta incertidumbre en las tesis, así que, al momento de realizar un proyecto de ese calibre, es primordial un análisis moderado y específico para entender el comportamiento del agua que yace en el sector a estudiar. Los distintos análisis hidrológicos amplían la información y abren espacio a un mejor diagnóstico sobre las fuentes hídricas, de sectores en específico, como una ejemplificación a esto se puede ver las diferentes funciones que se generan mediante cálculos matemáticos que proporciona el tiempo de época seca o con mayor precipitación. También con Qgis, que con sus herramientas se puede entender el Modelo de Elevación digital (DEM) para ver con precisión la concentración de agua en la época seca, y sus flujos, que por escurrimiento del agua de precipitación, se crean y convergen.

El objetivo de este estudio es inicialmente realizar un diagnóstico de la productividad de la finca en términos de disponibilidad y aprovechamiento hídrico, pues de esta manera se podrán cuantificar, mediante métodos hidrológicos, los datos e información hidrometeorológica y geográfica con los diferentes sistemas de información geográficos a usar. Lo anterior con el fin de establecer parámetros que proporcionen la idealización correcta de la finca para la implementación de un sistema VAC caracterizado por ser un sistema de acuicultura integrada.

Para la implementación del sistema VAC se deben tener en cuenta distintos factores como las terrazas de huerto, estas se caracterizan por la conducción de agua lluvia hacia el cultivo mediante las pendientes del mismo, puesto que son franjas horizontales construidas de manera horizontal, el ancho de la plataforma está dado por la pendiente o por el ancho de la copa frutal que se maneje, asimismo por la profundidad de la capa del suelo, se recomienda el uso de un cultivo denso y de esta manera evitar la erosión del suelo [3]; corral de ganado, para el desarrollo del sistema VAC se debe tener un número determinado de vacas (16) ubicadas cerca al estanque de peces y de esta manera se usará como abono a base de estiércol para los árboles. Otro importante aporte de las reses en la finca es el alimenticio, que beneficia el ingreso económico de la finca, además de vender y consumir sus derivados [3]; estanque de peces, la explotación agrícola es característica por la combinación de varias actividades de producción que conllevan a la disminución del riesgo de fracaso agrónomo. En lo anterior radica la importancia de integrar la acuicultura, debido a las características de los animales acuáticos, que son de sangre fría y utilizan la energía para crecer rápidamente, con el consumo de alimentos naturales o industriales. Otras ventajas del estanque es la disminución del riego ya que hacen viable irrigar los cultivos y abrevar los animales durante las épocas de sequía, aparte de aumentar la disponibilidad de proteínas para el consumo familiar y generar ingresos económicos [4].

\section{OBJETIVO}

Elaborar una propuesta para mejorar la productividad de la finca El Caimo, ubicada en el municipio de Villa de Leyva, Boyacá, teniendo en cuenta la disponibilidad de agua lluvia y la optimización de los procesos.

\section{METODOLOGÍA}

1) En primer lugar, se realizó la consulta de los datos hidrometeorológicos por medio de la página del Ideam, Dhime, con la localización de la estación meteorológica más cercana al proyecto. Se determinó así el mes más seco y el más lluvioso en el municipio durante el periodo de tiempo del año 2000 a 2020.

2) Se procedió a cuantificar, mediante métodos hidrológicos, los datos de precipitación mensual y diaria en el periodo de 2000 a 2020. Estos se interpretaron con el manejo de Qgis como sistema de información geográfica (SIG), para que a partir de un modelo de elevación digital (DEM) del 
terreno se realice el proceso de delimitación de la cuenca cercana a la finca, con cálculo del índice de vegetación de diferencia normalizada (NDVI) y de la visualización de los flujos de agua con el manejo del programa SAGA, el software Qgis y Google earth explorer en el terreno, esto con el fin de comprender el comportamiento vegetal e hídrico. Posteriormente, con la utilización del método de número de curva $(\mathrm{CN})$ del Servicio de Conservación de Suelos (SCS) de 1986, se llevó a cabo una estimación de los valores de escorrentía e infiltración en función de las condiciones propias del suelo en términos de cobertura y usos de la tierra, valores que arrojan un diagnóstico en términos de disponibilidad y aprovechamiento hídrico en la finca, como también se calculó el tránsito de crecientes a partir de los datos de precipitación diaria para el análisis de datos correspondientes a las corrientes durante un lapso de tiempo de 24 horas, lo que genera una relación con el coeficiente de escorrentía a partir del tipo de suelo de la finca y el estado del uso de suelo que le corresponde al área forestal.

3) Por último, para el diseño del sistema VAC se recurrió al Manual básico de Agro-acuicultura integrada con el fin de realizar una distribución adecuada y favorable del sistema en la finca, en donde se determinó que este sistema contará con un huerto, un estanque de peces, una pocilga de cerdos y un corral de ganado, para mejorar el desarrollo de la producción en la finca con la integración del recurso hídrico de manera óptima, para el rendimiento de productos agrícolas y pecuarios de manera sostenible.

\section{RESULTADOS}

\section{A. Información hidrometeorológica}

Los datos promedio mensuales de precipitación del municipio de Villa de Leyva, en donde se encuentra ubicada la finca El Caimo, se tomaron de la estación Villa de Leiva - AUT [24015300], una de las estaciones cercanas a la finca y ubicada en Arcabuco, Boyacá. Datos tomados en el periodo 2000-2020, y que su última lectura en lo corrido del año fue la del mes de junio del año 2020, con lo cual se logró determinar el mes más lluvioso (julio) y el mes más seco (febrero) en el municipio durante el periodo de tiempo determinado, que coinciden con los meses de los períodos de lluvia y sequía, según determinaciones del Ideam para el departamento de Boyacá, donde el régimen de lluvias es variado. Al centro y occidente del departamento se observa un comportamiento bimodal, con una época seca principal al comienzo del año y una ligeramente menos marcada, a mediados de año. Las temporadas de lluvia en estas dos zonas tienen lugar en los meses de marzo-mayo y septiembre-noviembre. Al oriente del departamento, el régimen es el característico de la Orinoquia, con una única estación seca de diciembre a marzo, y una temporada lluviosa que se prolonga durante el resto del año con máximos de lluvia en los meses de junio-julio [5].

Para una mayor representación del régimen de los datos mensuales de precipitación se presenta el siguiente gráfico:

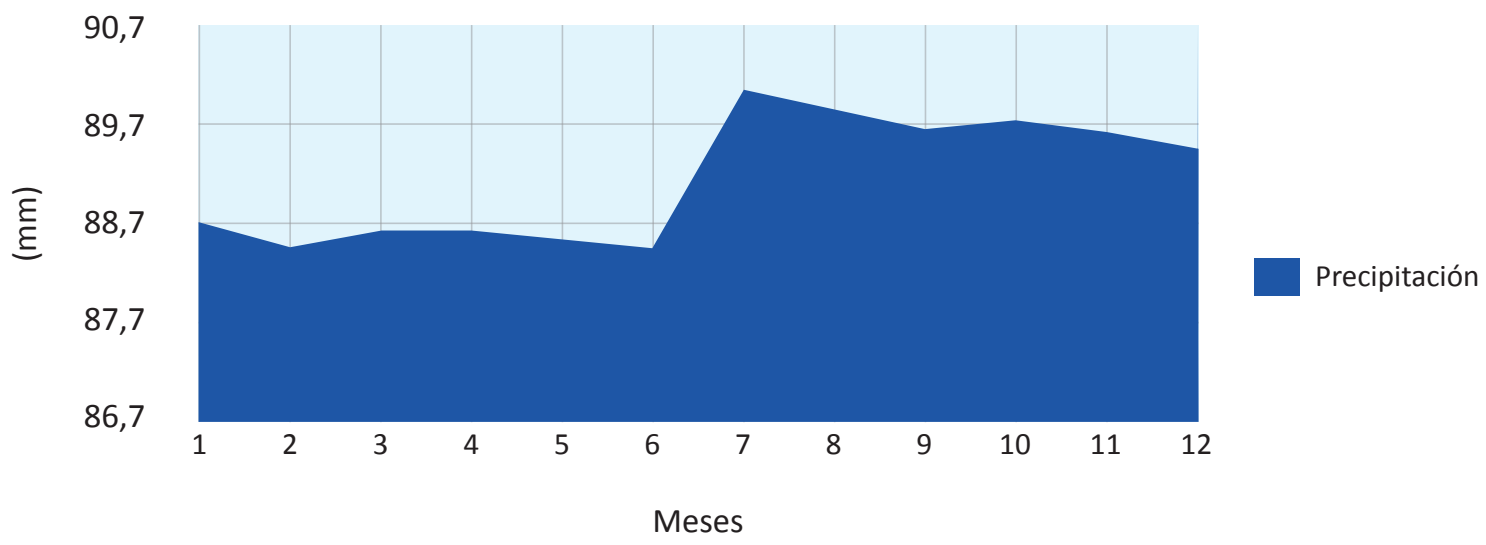

Figura 1. Promedio de precipitación mensual en el periodo 2000-2020, del municipio de Villa de Leyva, Boyacá. Fuente: elaboración propia. 


\section{B. Cálculo del índice de vegetación de diferencia normalizada (NDVI)}

Principalmente, para poder comprender los flujos de agua que hay en un sector se debe definir el lugar a estudiar. Por ende, a través del sitio web
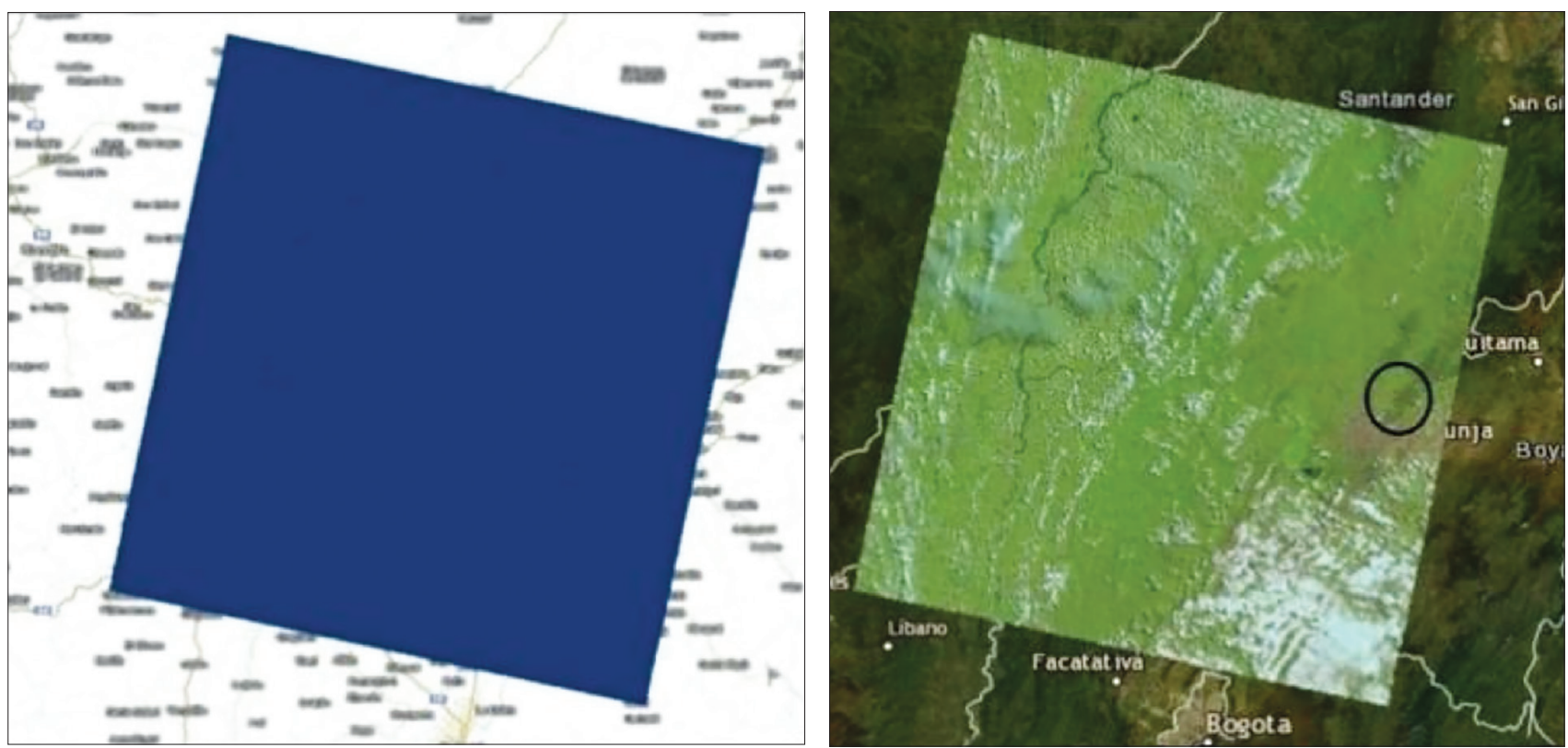

Google earth explorer, el cual permite obtener un modelo digital de elevación (DEM), se optó por la descarga de una imagen que cubriera Villa de Leyva en la época seca y posteriormente hacerle modificaciones en el programa Qgis, para entender el comportamiento de los cuerpos de agua y la vegetación en esta época del año.

Figura 2. Comparación de imágenes trabajadas con respecto a los flujos de agua, izquierda (polígono con cambios en Qgis) y derecha (DEM).

Fuente: elaboración propia.

En la figura 2 se evidencia una comparación entre la imagen DEM, y la misma imagen, pero ya con los cambios que se realizaron en Qgis. El circulo negro de la imagen DEM marca la ubicación de la finca para facilitar su localización en la comparación de las imágenes. En la parte izquierda, específicamente en el polígono con los cambios de Qgis, se pueden observar pequeñas hebras blancas que representan los cuerpos de agua que pasan por el polígono georreferenciado de Villa de Leyva y Tunja. Asimismo, si se analiza por los lados de la finca, estas hebras no tienen mayor presencia, lo que infiere que por la finca no hay gran ración hídrica que puedan abastecer certeramente al Caimo. Además, estas modificaciones de Qgis también se hicieron en el mismo polígono, pero con el fin de entender la parte vegetativa que hay por el lugar a estudiar, esto con el objetivo de comprender cómo la 
La delimitación de la cuenca es sustancial, pues ayuda a buscar los escurrimientos del sector, o como su nombre expone, de la cuenca. Se generó la delimitación que se observa en la figura 4 , para que trabaje en sinergia con el cálculo de los flujos y poder tener un conjunto más preciso de información sobre el comportamiento de los cuerpos de agua que pasan específicamente por el polígono en donde se encuentra ubicada nuestra finca. Todo esto con el fin de entender y aplicar un sistema VAC capacitado para mantener la supervivencia de la finca El Caimo.

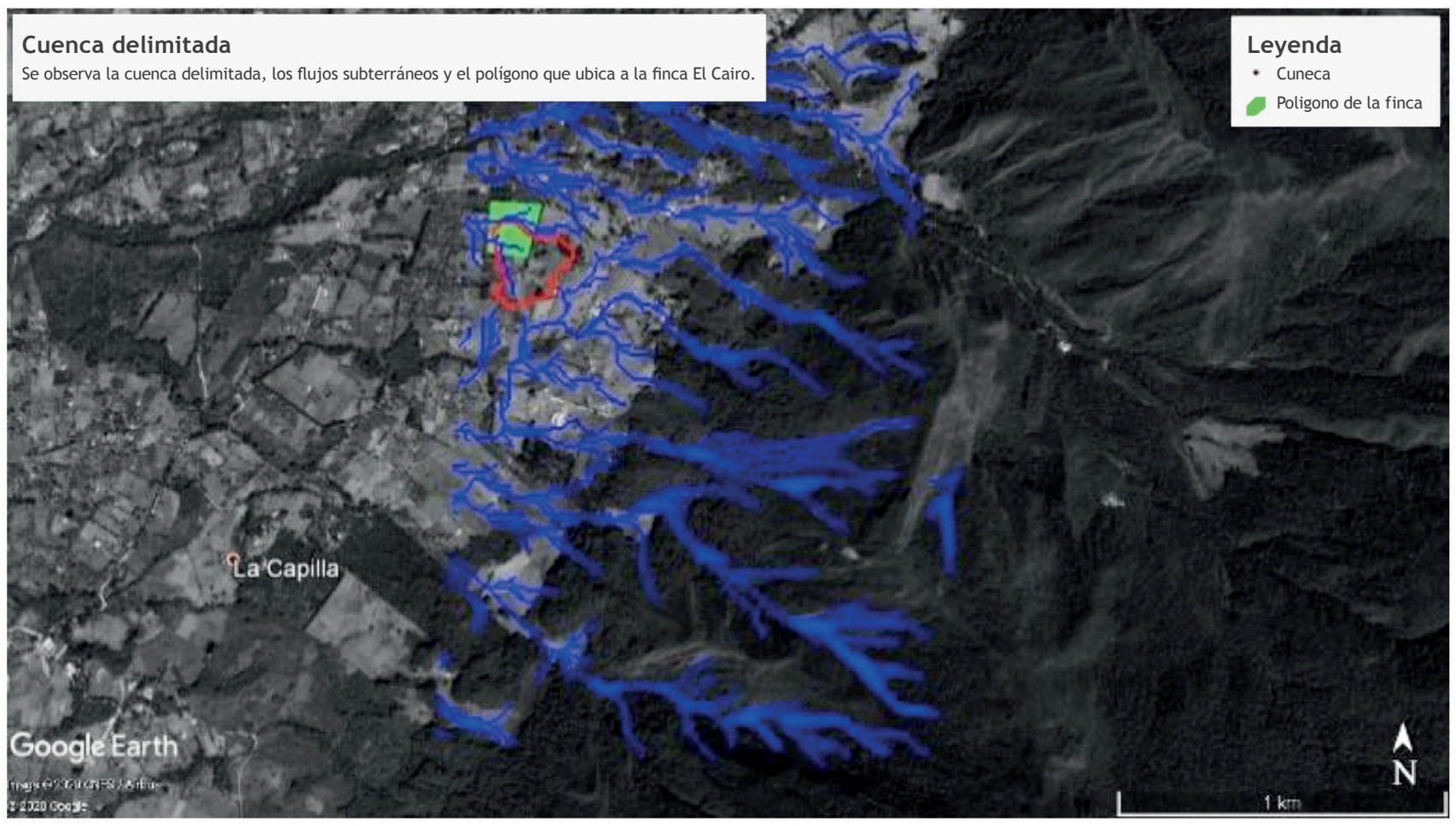

Figura 3. Cuenca delimitada con respecto al área de interés.

Fuente: elaboración propia.

\section{Método del número de curva $(\mathrm{CN})$}

Se utiliza el método de número de curva del servicio de Conservación de Suelos del Departamento de Agricultura de los Estados Unidos (SCS) de 1986 actualmente Servicio de Conservación de Recursos Naturales (NRCS) de Estados Unidos, para realizar una estimación de los valores de escorrentía en función de las condiciones propias del suelo según el tipo o grupo suelo C (margas arcillosas, margas arenosas poco profundas, Suelos de bajo contenido orgánico y Suelos generalmente altos en arcilla) con su clasificación (mala, regular y buena) referida a su condición hidrológica, según uso/ tratamiento de la tierra, y densidad a partir de interpretación visual de imágenes satelitales [6]. Para determinar el desarrollo productivo actual de la finca El Caimo en términos de oferta y captación de agua lluvia se ha de tener en cuenta que en principio la finca cuenta con un tipo o grupo de suelo $C$, al que se le añade la variable de la cobertura del predio y su clasificación acorde a la condición en la que se encuentra, que se representa en: árboles (mala), cultivos en línea (mala) y tierra sin cultivar (buena) con sus respectivos CN (cantidad de agua superficial en \%) tomados de la tabla 1 y representados en la tabla 2 con respecto al área total de la finca $33,461 \mathrm{~m}^{2}$, con el empleo de la herramienta Google earth y saberes previos de la finca.)

Determinación del número de curva ponderado (potencial de escorrentía) para la cuenca en su conjunto con un $23 \%$ de árboles, $19 \%$ de cultivos en línea y un $58 \%$ de tierra sin cultivar aproximadamente, como se muestra en la tabla 2. 


\begin{tabular}{|l|c|c|}
\multirow{2}{*}{\multicolumn{1}{c|}{ Uso de la tierra }} & \multicolumn{2}{c|}{ Grupo de suelo C } \\
\cline { 2 - 3 } & Condición hidrológica \\
\cline { 2 - 3 } & Mala & Buena \\
\hline Árboles & 77 & 70 \\
\hline Cultivos en línea & 88 & 85 \\
\hline Contorno y terrazas & 80 & 78 \\
\hline Tierras agrícolas, pastos o praderas no cultivadas & 86 & 74 \\
\hline
\end{tabular}

Tabla I. Algunos usos de la tierra para grupo hidrológico de suelo c Fuente: elaboración propia.

\begin{tabular}{c|c|c|c|c|}
\multicolumn{6}{c|}{ Cobertura actual } \\
\hline $\begin{array}{c}\text { Grupo } \\
\text { de suelo }\end{array}$ & Uso & Área & $\begin{array}{c}\text { Porcentaje } \\
\text { de ocupación }\end{array}$ & $\begin{array}{c}\text { Número de curva } \\
\text { (condición hidrológica) }\end{array}$ \\
\hline \multirow{2}{*}{ C } & Árboles & 7,606 & 22.73 & 77 \\
& Cultivos en línea recta & 6,260 & 18.71 & 88 \\
\hline
\end{tabular}

Tabla 2. Valores de escurrimiento acordes a la cobertura existente, su condición hidrológica y grupo de suelo Fuente: elaboración propia.

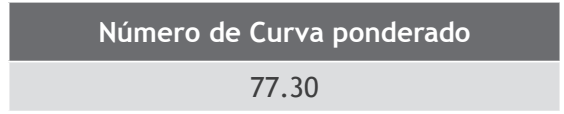

Tabla 3. Número de curva actual de la finca El Caimo.

Fuente: elaboración propia.

Con lo cual se desea generar una mayor cobertura vegetal con la especie endémica de roble andino (Quercus humboldtii) apoyada en la siembra de cultivos que frutales (mora, durazno) y hortalizas (papa) que se producen actualmente en la finca, y una cobertura con una condición hidrológica buena a raíz de la diferencia en sus valores de escorrentía (tabla 4) para un grupo de suelo $\mathrm{C}$ y representado en la figura 4 . Pensando en disminuir la escorrentía superficial al cubrir de árboles una zona poco cubierta y a su vez de cultivos en forma de terrazas, un $17 \%$ del agua lluvia que cae deja de escurrir en la superficie acorde a la tabla 4, con el control de los niveles de escorrentía, y evitando pérdidas de agua por erosión hídrica que generan daños en los suelos y en los cauces próximos por crecidas abruptas de los niveles del agua durante el período de lluvias [7].

\begin{tabular}{|c|c|}
\hline \multicolumn{2}{|c|}{ Escurrimiento } \\
\hline Usos & Diferencia (\%) \\
\hline Árboles & 7 \\
\hline Cultivos & 10 \\
\hline Total & 17 \\
\hline
\end{tabular}

Tabla 4. Diferencia de escurrimiento al cambiar la condición hidrológica de los usos de la tierra de la cobertura de la finca Fuente: elaboración propia. 


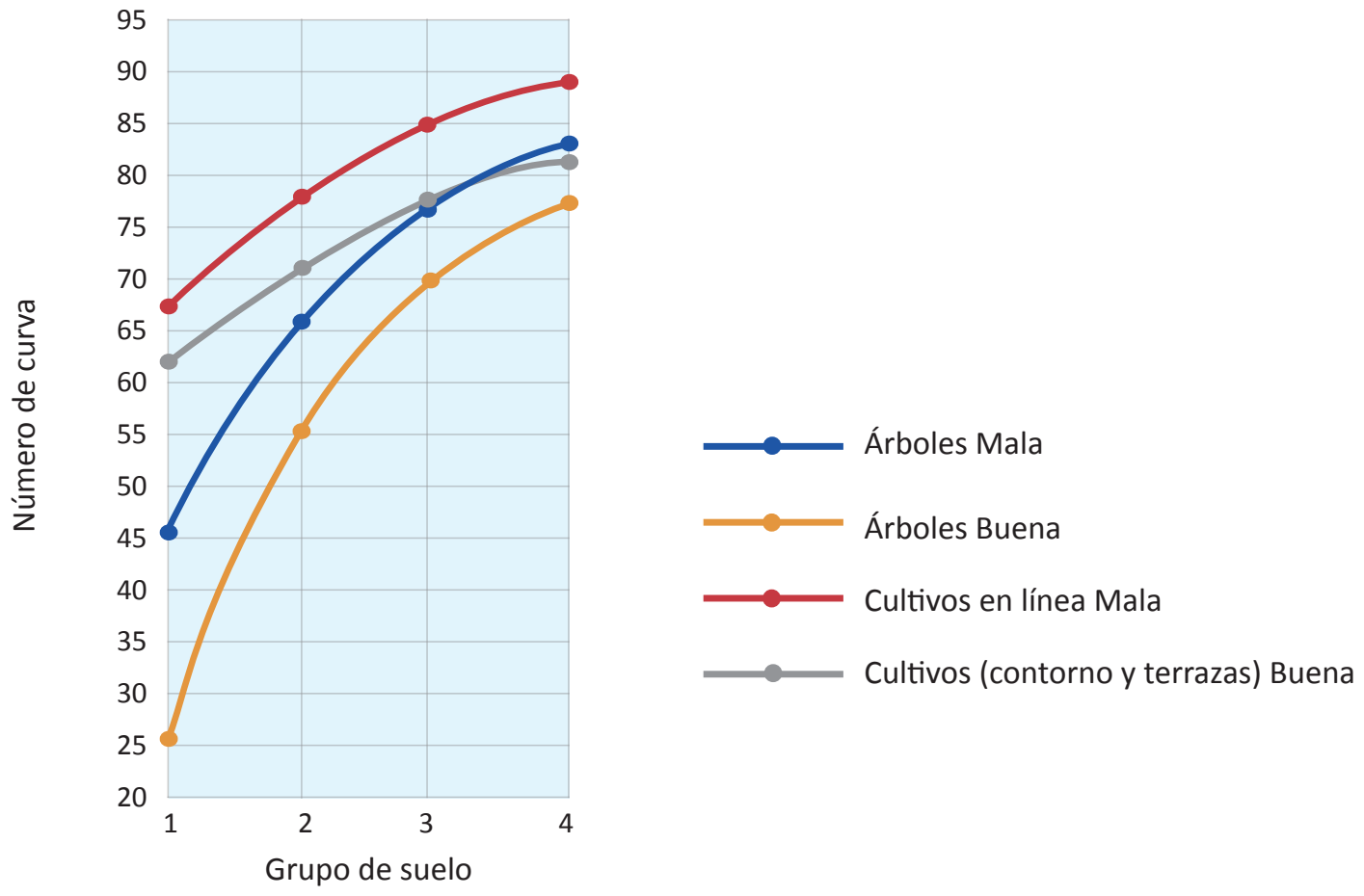

Figura 4. Comparación de escurrimiento del cambio en la cobertura para árboles y cultivos según número de curva (CN) y grupo de suelo 1 (A), 2 (B), 3 (C), 4 (D).

Fuente: elaboración propia.

Con la realización de una mejora en el desarrollo productivo actual de la finca a partir de la captación de la descarga hídrica por escurrimiento de la cuenca conforme al tipo o grupo de suelo $\mathrm{C}$ de la finca y su cobertura, se realiza un cambio en el área forestal y de cultivos junto con el área de la finca sin cultivar, con una plantación de la especie roble andino (Quercus humboldtii) y la siembra de cultivos de papa, mora y durazno en forma de terrazas o plataformas horizontales con cierta contrapendiente en la ladera, según curvas de nivel, en la sección disponible para ello, como lo es el área sin cultivar y parte de la que ocupan actualmente los cultivos en línea que se encuentran establecidos en la finca y que se pretenden levantar, con el fin de adecuar la cobertura según la convergencia de los flujos de agua lluvia en el terreno.

Por lo tanto, se determina el nuevo número de curva (potencial de escorrentía) (tabla 5), y con dicha mejora que indica una disminución de un 4 $\%$ del número de curva ponderado.

\begin{tabular}{c|c|c|c|c}
\multicolumn{6}{c}{ Nueva cobertura } \\
\hline $\begin{array}{c}\text { Grupo } \\
\text { de suelo }\end{array}$ & Uso & Área & $\begin{array}{c}\text { Porcentaje de } \\
\text { ocupación (\%) }\end{array}$ & $\begin{array}{c}\text { Número de curva } \\
\text { (condición hidrológica) }\end{array}$ \\
\hline \multirow{2}{*}{ C } & Árboles & 13,391 & 40.02 & 70 \\
& Cultivos (contorno y terrazas) & 5,522 & 16.50 & 78 \\
\hline
\end{tabular}

Tabla 5. Valores de escurrimiento acorde a la mejora en la cobertura, su condición hidrológica y grupo de suelo. Fuente: elaboración propia.

\section{Nuevo número de curva ponderado}

73.06

Tabla 6. Nuevo número de curva ponderado para la finca El Caimo Fuente: elaboración propia. 


\section{E. Infiltración de agua lluvia}

A la hora de determinar la capacidad de infiltración del suelo de la finca se tuvo en cuenta las variables de las que depende, como del tipo de cubierta vegetal que corresponde a árboles de las especies pino (Pinus), eucalipto (Eucalyptus), aliso (Alnus acuminata), la especie que forma parte de la propuesta roble andino (Quercus humboldtii) junto con la siembra de cultivos de papa, mora y durazno, como también de las características del suelo que acorde con el número de curva ponderado (CN) corresponde al grupo de suelo C (margas arcillosas, margas arenosas poco profundas, suelos de bajo contenido orgánico y suelos generalmente altos en arcilla), también de la cantidad de agua lluvia mensual según los datos de precipitación del municipio de Villa de Leyva, Boyacá representados en la figura 1, el valor del área de captación (cuenca) de 60,712 $\mathrm{m}^{\wedge} 2$ delimitada, y el coeficiente de escorrentía para el $100 \%$.

Inicialmente se determina la diferencia en la infiltración con la cobertura actual y cobertura nueva, propuesta en la figura 5 , en donde la cobertura actual alcanza una recepción de 1,257,183 L de agua al mes, que difieren del 1,475,823 L de agua que se obtendrían con la cobertura nueva, es decir de un aumento del $17 \%$ de la recepción actual de agua. Lo que arroja resultados positivos en la mejora de la cobertura de la finca en conjunto.

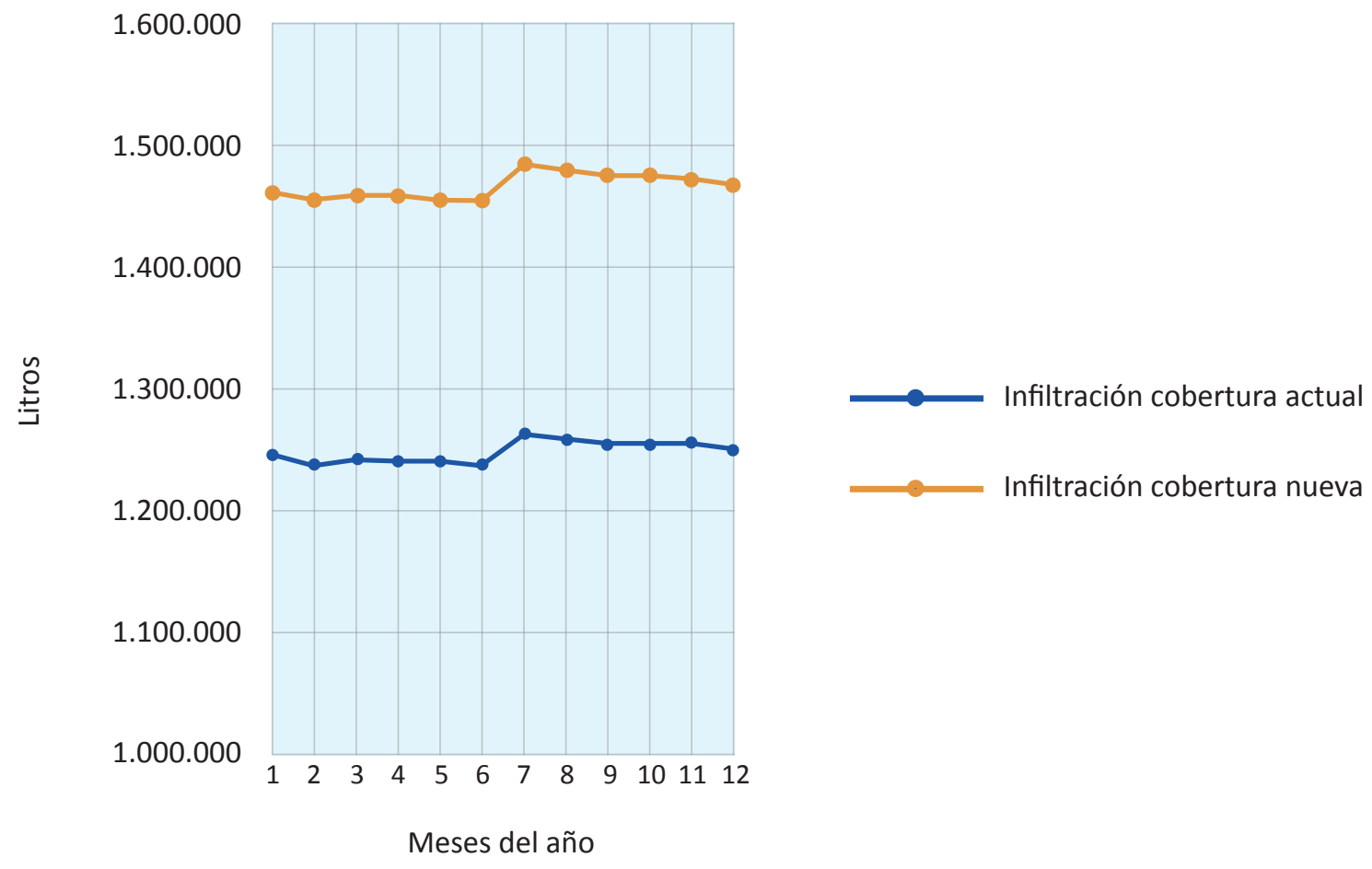

Figura 5. Diferencia de escenarios de infiltración según la cobertura de la finca El Caimo en el municipio de Villa de Leyva, Boyacá.

Fuente: elaboración propia.

\begin{tabular}{|c|c|c|}
\hline \multicolumn{3}{|c|}{ Infiltración de la cobertura } \\
\hline Área & Max & Min \\
\hline Actual & $1,257,183$ & $1,234,947$ \\
\hline Nueva & $1,475,823$ & $1,449,720$ \\
\hline
\end{tabular}

Tabla 7. Litros de agua mensual aprovechables según la cobertura de la finca El Caimo Fuente: elaboración propia. 
Reconociendo el valor agregado que traería el aumento del área forestal con la plantación de árboles de roble andino (Quercus humboldtii) y la mejora en la adecuación del cultivo en forma de terrazas como sistema de conservación del recurso hídrico, ya que disminuyen la velocidad del agua de escorrentía en la ladera, aumentan la capacidad de infiltración, limitan el arrastre del suelo y evacuan el agua sobrante en la ladera, lo que facilita las operaciones de plantación y siembra (repoblación) para la finca El Caimo, con la disminución del riego en época seca al conservar el agua de la época de lluvias de forma subterránea, ocasionando la máxima infiltración y almacenaje de agua a través de la raíces de los árboles en los acuíferos subterráneos, y por su acción de enfriamiento durante su proceso de evapotranspiración, con la regulación del ciclo hidrológico, ya que al ser una especie maderable cuenta con una mayor tasa de evapotranspiración [8], mantiene la humedad, mejora la fertilidad del suelo y aumenta la fijación de nitrógeno atmosférico [9].

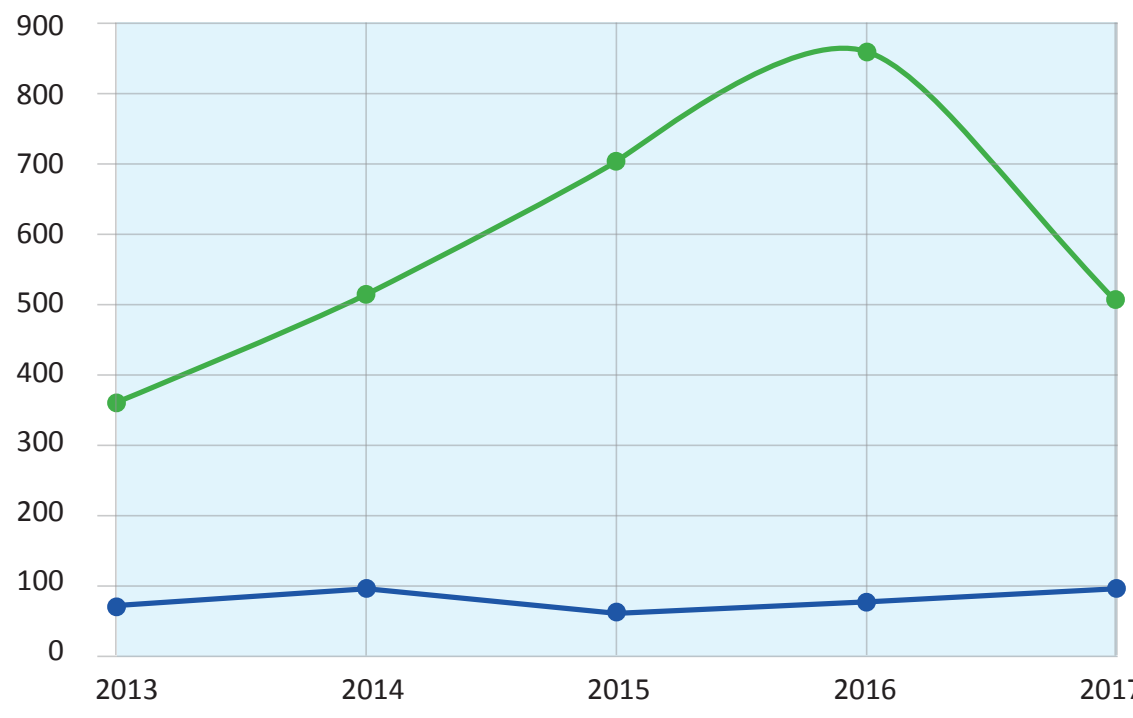

Figura 6. Relación de la deforestación con los niveles de precipitación en el departamento de Boyacá.

Fuente: elaboración propia.

Siembra a raíz de la tendencia de la deforestación en el departamento a razón de la precipitación promedio anual del municipio de Villa de Leyva.

A partir de la figura 6, se evidencia el efecto de retroalimentación entre los procesos tierra-atmósfera, y se demuestra que las pérdidas de agua provocadas por la evapotranspiración son fundamentales para mantener el balance del régimen hidrológico. La deforestación reduce los caudales medios y aumenta los caudales extremos con efectos en inundaciones y sequías más fuertes y más frecuentes. Además, la deforestación provoca aumentos de temperatura superficial, aumento de presión atmosférica, disminución en la humedad del suelo, disminución en la evapotranspiración, aumento del albedo, disminución de la cobertura de nubes y las lluvias en el mediano y en el largo plazo con la disminución de los caudales medios de los ríos [10]. Aunque el mayor valor de deforestación registrada 854 ha en el departamento de Boyacá en el periodo de 2016-2017 [11] no resulta tan significativo en comparación con las 200000 km²de deforestación de los bosques tropicales que se presentan cada año; son semejantes en los objetos de su actividad como el convertir las tierras en cultivos, para el pastoreo de ganado o para la extracción de madera [10]. Siendo esto lo que convierte a los árboles en protectores de la vegetación adyacente, la fauna, y en reguladores del régimen hidrológico y del clima. 


\section{F. Cálculo de tránsito de crecientes}

Determinación del punto máximo de almacenamiento de agua lluvia que indica la creciente respecto a infiltración y la disminución de escorrentía en la finca.

Se realizó el análisis de datos correspondiente al tránsito de corrientes de la finca durante un lapso de tiempo de 24 horas tomado del promedio de precipitaciones previamente revisados en la información de la estación Villa de Leiva - AUT [24015300] siendo esta la más cercana a la finca y ubicada en Arcabuco, Boyacá. Lo anterior genera una relación con el coeficiente de escorrentía a partir del tipo de suelo de la finca. El hidrograma del gráfico $x$ genera específicamente la relación entre una cobertura del suelo mala y buena, pero se prioriza la condición que toma el máximo nivel de agua acumulada con la cobertura arbórea al 100 \% como caso hipotético, a partir del cual se determinarán las mejoras a las condiciones actuales respecto a la infiltración de la finca que aumentará, dando paso a un escurrimiento de agua lluvia menor.

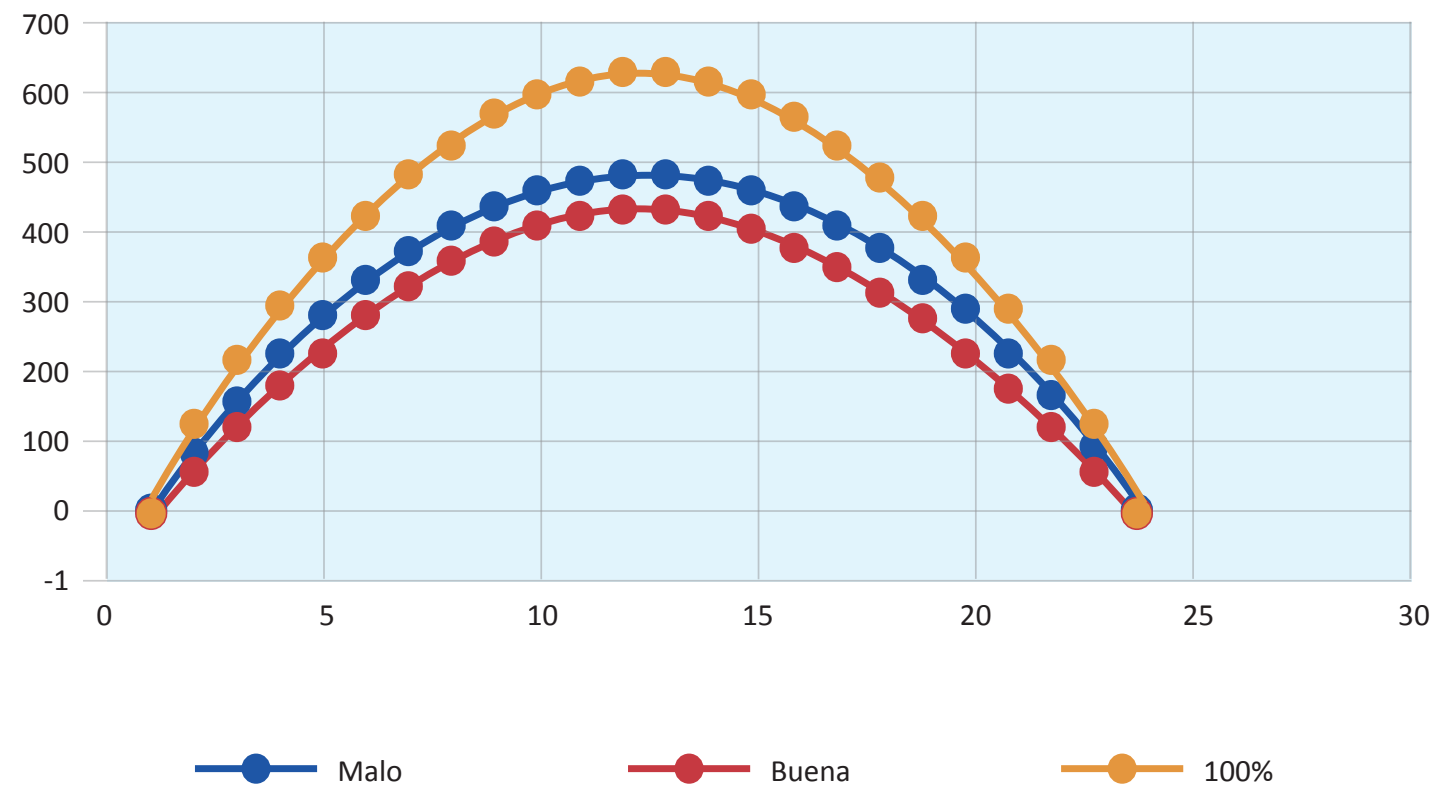

Figura 7. Hidrograma comparativo para la cobertura de árboles según su condición hidrológica. Fuente: elaboración propia.

El tránsito de crecientes relacionado a la finca se tomó realizando una mejora en la cobertura arbórea mediante surcos y medido a partir del método de embalses lineales, el cual permite relacionar su almacenamiento con el caudal de salida, dado este a partir de una definición algebraica se permite realizar el análisis volumétrico y del caudal o tasa de flujo a partir de la constante de almacenamiento en específico [12].
En la figura 8, además se evidencia un punto central entre las coberturas buena y mala, donde permite reconocer el almacenamiento y función de distribución dada a partir de las mejoras, siendo esta la diferencia entre las coberturas buena y mala, tomando el valor de máximo caudal en el día. Hora:12 p. m. / Cm. Cobertura mala $=4.819 / \mathrm{Cb}$. Cobertura buena $=4.391$. De modo que, la disminución del caudal que va al acuífero será $\mathrm{Q}=\mathrm{Cm}-\mathrm{Cb}$. 
$0,428 \mathrm{~m}^{3} / \mathrm{h}$ lo que disminuye mayoritariamente las posibilidades de inundación en la vereda Capilla 2 y asimismo mejora las capacidades de infiltración de la finca para la adaptación al sistema VAC. Por lo tanto, la modelación hidrológica resultante permite determinar las diferentes condiciones a las que se expone el agua subsuperficial estudiada, de tal manera que se genera un comparativo de entrada con la cobertura arbórea y también acerca de sus áreas impermeables y permeables respecto a la cuenca donde está ubicada la finca, e indica principalmente la diferencia de valores respecto al transporte del caudal como a las posibilidades de mejora [12].

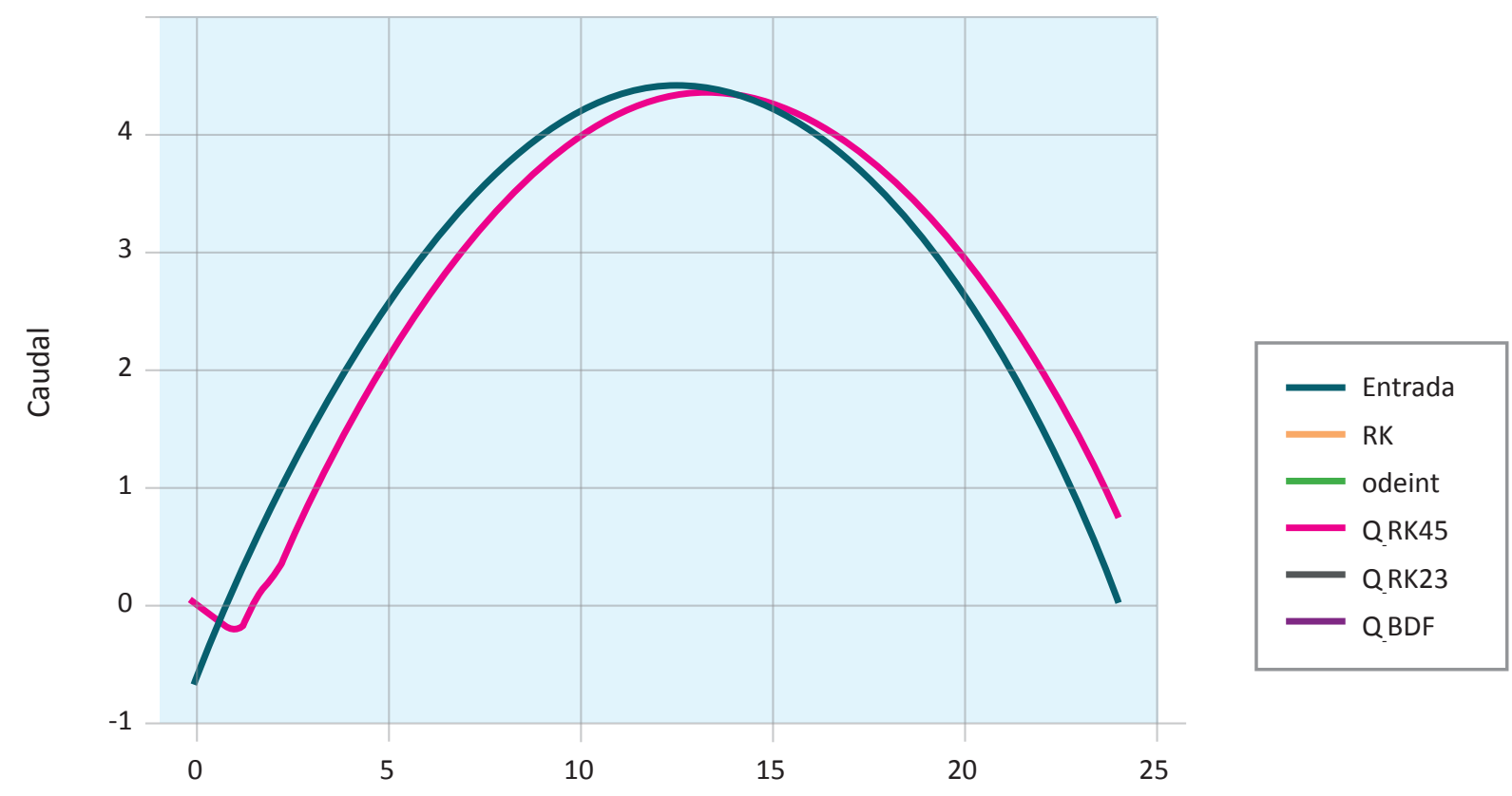

Horas

Figura 8. Hidrograma del caudal acumulativo entre una condición hidrológica mala y buena para uso de árboles en el terreno.

Fuente: elaboración propia.

\section{G. Sistema VAC}

1) Sistema integrado de cultivo en tierras altas

- Terrazas de huerto

Se establece la siembra de cultivos frutales como mora y durazno, y hortalizas como la papa, el área total de la terraza en la finca El Caimo se determinaron $5,522 \mathrm{~m}^{2}$ construido bajo alturas del banco que no sobrepasen los $2 \mathrm{~m}$ de altura.

Para la fertilización de los cultivos frutales se ha de emplear el lodo del fondo del estanque de peces que se saca cada 3 a 4 años, para el cultivo de hortalizas (papa) se abonan según sea necesario [3] y para la plantación de árboles roble andino (Quercus humboldtii.) y demás especies presentes en la finca Él Caimo, sin distinción se abonan una o dos veces al año con el uso del estiércol del ganado, este último es un fertilizante por excelencia y se le atribuye su alto contenido de nitrógeno, y en materia orgánica restaura los niveles de nutrientes del suelo, los cuales dependen del tipo de ganado y su alimento, teniendo en cuenta que este no se aplica de manera directa a los cultivos sino mucho antes de la siembra, regulando sus dosis para evitar la contaminación de aguas superficiales o subterráneas por nitratos de origen agrícola [13]. 


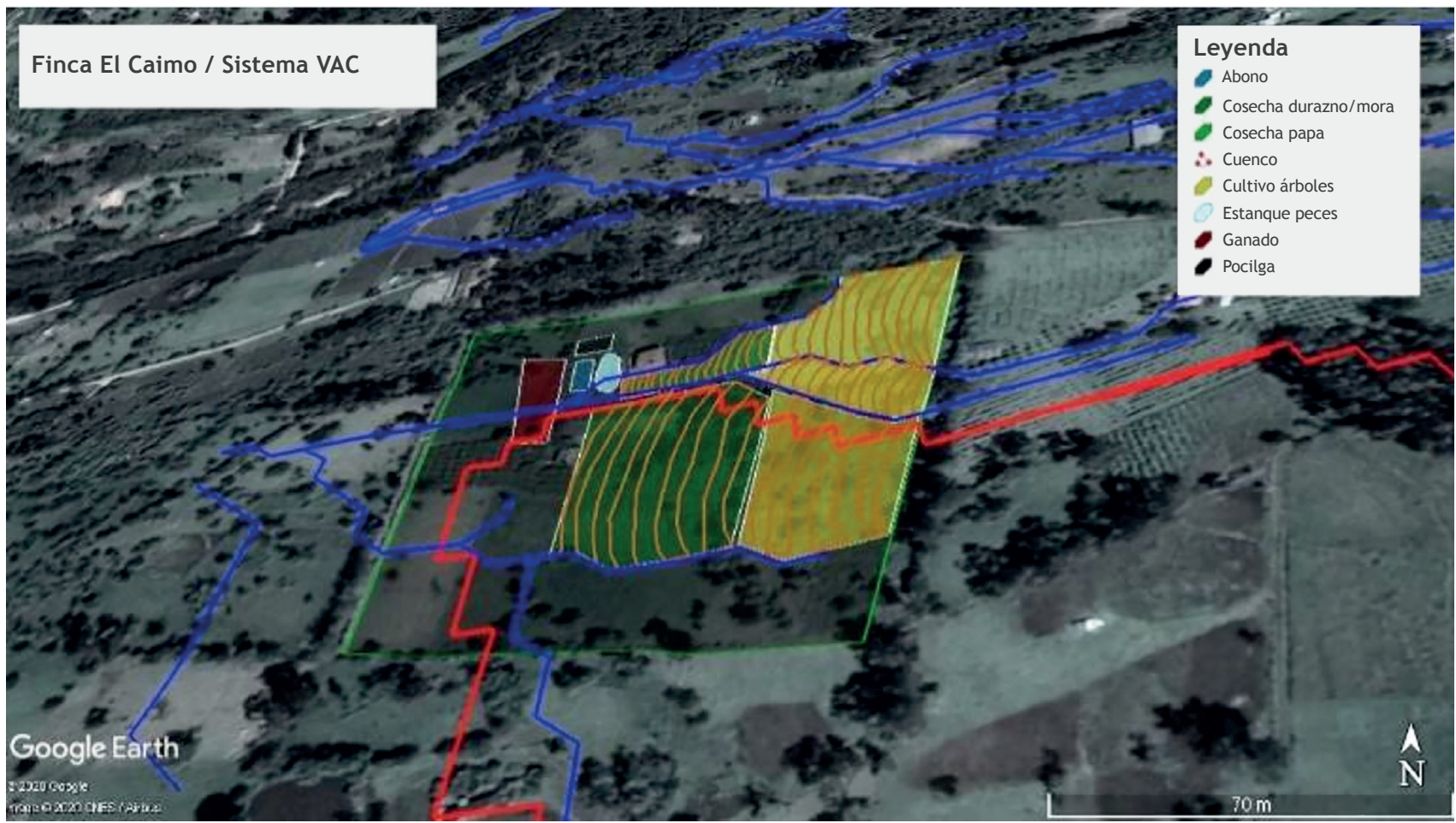

Figura 9. Sistema VAC para la finca El Caimo.

Fuente: elaboración propia.

\section{- Corral de ganado}

La adecuación de vacas en la finca se convierte en algo beneficioso, pues si la manutención es reducida al alimento del pastoreo y los subproductos de la finca, se mejoran los cuidados de manera significativa [3].

\section{- Pocilga de cerdos}

La pocilga de cerdos funciona en el sistema VAC para la limpieza de los desechos orgánicos y los residuos de otros subproductos de la granja, como papa, mora y durazno (cultivos del huerto). Pues estos desperdicios se usan para su alimentación a base de lavaza (mezcla de desperdicios orgánicos), siendo estos animales de gran ayuda para la sostenibilidad de la finca, además de poder utilizar su estiércol como abono para el huerto o los árboles, y para el estanque de peces.

El número de cerdos estimado es de 1-3 según el Manual básico de Agro-acuicultura integrada, teniendo en cuenta la cerda principal de cría para la reproducción de otros cerdos. Es importante mencionar que la crianza de cerdos también aporta ganancias económicas para la finca si se venden como producto alimenticio o simplemente para otras personas del campo.

\section{- Estanque de peces}

El estanque propuesto para la finca El Caimo cuenta con un área de $162 \mathrm{~m}^{2}$ y $1 \mathrm{~m}$ de profundidad, a su vez tres especies característicamente relevantes para el sistema VAC como en el mismo porcentaje (\%) de ocupación; la trucha arcoiris, importante para el desarrollo económico del sistema y como suministro de proteína, esta especie se alimenta principalmente de insectos y moluscos; Tilapia Rendalli, la cual aporta beneficios como la respiración aérea adecuada para la adaptación a circunstancias difíciles debido al deterioro gradual del agua en sequías y su consumo herbívoro que asegura la limpieza del estanque con el consumo de la cobertura biológica y macrófitas; la Carpa Plateada, que cumple la misma función herbívora y además consume detritos, fitoplancton y zooplancton [14].

De otro lado, el mantenimiento y función del estanque es gracias a su ubicación cercana a la casa que facilitará la disposición de desechos orgánicos, siendo estos la principal fuente del lodo que será extraído cada 3 o 4 años como abono [3]. 


\section{CONCLUSIONES}

- Es necesario reevaluar si el modo en el que se está produciendo el desarrollo de la finca es el adecuado según las características del suelo, las condiciones climáticas y la dinámica hidrológica, para así determinar los factores que limitan o potencian el uso y manejo del agroecosistema, como el tipo de cobertura, los usos del suelo, la disponibilidad hídrica, la topografía del lugar, entre otros.

- En el análisis expuesto de los cálculos NDVI que ayudan a entender los flujos de agua y la vegetación en época seca, podemos comprender la importancia de los flujos de agua subterráneos, que son el soporte para que la flora del sector de la finca no se vea en riesgo. Además, los flujos subterráneos apoyan el índice hídrico de la finca en épocas precarias.

- Con el cambio en la cobertura actual de la finca El Caimo se obtiene una disminución del 4 $\%$ en el potencial de escorrentía y un aprovechamiento de 1,475,823 L de agua de infiltración. Asimismo, el cálculo de disminución del caudal debido a la nueva cobertura arbórea en la finca indicó una reducción de $0,428 \mathrm{~m}^{3} / \mathrm{h}$, aportes hidrológicos que resultan significativos en la implementación del sistema VAC y sus componentes.

- Es necesario el empleo de herramientas tecnológicas de modelación hidrológica y del terreno, para mejorar las condiciones productivas de la finca adecuando los componentes del sistema de acuerdo a eso y en miras a la conservación del recurso hídrico y del suelo.

- El agua señala ser la principal fuente de abastecimiento para el consumo y las labores de la finca, de ahí la necesidad del cambio en la cobertura para aumentar la recarga de acuíferos subterráneos para su reserva en el subsuelo y poder mantener hidratado el suelo en temporadas de sequía.

- En definitiva, la adopción de un sistema VAC genera mayor rentabilidad a través de la conexión complementaria de las distintas actividades que se desarrollan dentro de la finca, que conforman su estructura, y de las cuales emergen las salidas del sistema, que defienden en gran parte su funcionalidad.

\section{REFERENCIAS}

[1] S. Fatorelli y F. Pedro, Diseño hidrológico. Zaragoza, Estudio Fernández-Dorca, 2011.

[2] W. Sandoval-Erazo, Diseño de obras hidrotécnicas. Sangolqui, Ediespe, 2018.

[3] Organización de las Naciones Unidas para la Alimentación y la Agricultura, Instituto Internacional para la Reconstrucción Rural, Worldfish Center, Agro-acuicultura integrada. Manual básico. Roma, FAO/ICLARM/IIRR, 2004.

[4] M. Halwart, M. Espinoza-Martínez y A. Schuckler, Los pequeños estanques, Roma, FAO, 2000.

[5] Atlas del Ideam - Boyacá, 2010. [En línea]. Disponible en: http://atlas.ideam.gov.co/ basefiles/boyaca_texto.pdf. [Accedido: 22-julio-2020].

[6] L. W. Mays, Ingeniería de recursos hídricos, 3a edición. New York, Wiley, 2019.

[7] Organización de las Naciones Unidas para la Alimentación y la Agricultura, Fondo Internacional para el Desarrollo de la Agricultura, y Cooperación Suiza en América Central, Captación y almacenamiento de agua de lluvia. Opciones técnicas para la agricultura familiar en América Latina y el Caribe. Santiago de Chile, FAO, 2013.

[8] G. A. Forero Buitrago, "La madera colombiana, oportunidad de regeneración del flujo de los ríos mediante una producción sostenible y competitiva", Rev. Tecnol., vol. 15, no. 2, pp. 113-128, 2017. doi: 10.18270/rt. v15i2.2179.

[9] E. Gras, Cosecha de agua y tierra. México, Coas ediciones, 2009.

[10] G. Poveda and O. Mesa, "Efectos hidrológicos de la Deforestación," ResearchGate, Dec. 1994. https://www. researchgate.net/ publication/254258616_Efectos_hidrologicos_de_la_Deforestacion. [Accedido: 3-diciembre-2020].

[11] Instituto de Hidrología Meteorología y Estudios Ambientales, "Sistema de Monitoreo de Bosque y Carbono," 2017. http://smbyc. ideam.gov.co/MonitoreoBC-WEB/pub/reporteGeoproceso.jsp?id_reporte $=7101$ [Accedido: 3-diciembre-2020]. 
[12] E. Ruiz y M. Miren, OpenCourseWare, [En línea]. Disponible en: https://ocw.ehu.eus/ pluginfile.php/4576/mod_resource/content/1/Material_Docente/Tema_5.pdf. [Accedido: 22-julio-2020].

[13] G. Tortosa, "Uso del estiércol como fertilizante”. Compostando Ciencia Lab., 2014. [En línea]. Disponible en: http://www.com- postandociencia.com/2014/08/uso-estiercol-como-fertilizante/. [Accedido: 22-julio-2020].

[14] A. Tacon, Nutricion y alimentación de peces y camarones cultivados. Manual de capacitación. Brasilia, FAO, 1989. [En línea]. Disponible en: http://www.fao.org/3/ab492s/ AB492S00.htm\#TOC 